ESAIM: COCV 27 (2021) 97

https://doi.org/10.1051/cocv/2021096
ESAIM: Control, Optimisation and Calculus of Variations

www.esaim-cocv.org

\title{
ANISOTROPIC MEAN CURVATURE FLOW OF LIPSCHITZ GRAPHS AND CONVERGENCE TO SELF-SIMILAR SOLUTIONS*
}

\author{
A. Cesaroni ${ }^{1, * *} \oplus$, H. Kröner ${ }^{2}$ And M. Novaga ${ }^{3} \odot$
}

\begin{abstract}
We consider the anisotropic mean curvature flow of entire Lipschitz graphs. We prove existence and uniqueness of expanding self-similar solutions which are asymptotic to a prescribed cone, and we characterize the long time behavior of solutions, after suitable rescaling, when the initial datum is a sublinear perturbation of a cone. In the case of regular anisotropies, we prove the stability of selfsimilar solutions asymptotic to strictly mean convex cones, with respect to perturbations vanishing at infinity. We also show the stability of hyperplanes, with a proof which is novel also for the isotropic mean curvature flow.
\end{abstract}

Mathematics Subject Classification. 53C44, 35K93 .

Received June 7, 2021. Accepted September 26, 2021.

\section{INTRODUCTION}

We consider the evolution of sets $t \mapsto E_{t}$ in $\mathbb{R}^{N+1}$ governed by the geometric law

$$
\partial_{t} p \cdot \nu(p)=-\psi(\nu(p)) H_{\varphi}\left(p, E_{t}\right)
$$

where $\nu(p)$ is the exterior normal at $p \in \partial E_{t}, \psi$ is a positive, continuous, 1-homogeneous function representing the mobility, $\varphi$ is a norm representing the surface tension, and $H_{\varphi}(p)$ is the anisotropic mean curvature of $\partial E_{t}$ at $p$, associated with $\varphi$, see Definition 2.1. This evolution is an analogue of the classical (isotropic) mean curvature flow, which corresponds to the case $\varphi(x)=\psi(x)=|x|$ and it is studied as model of crystal growth, see $[6,7,14,15]$. Existence and uniqueness of the level set flow associated to (1.1) have been obtained for general mobilities $\psi$ and purely crystalline norms $\varphi$ in [14, 15], in the viscosity setting, whereas the case of general norms $\varphi$ with convex mobilities $\psi$ has been treated in $[6,7]$, in the distributional setting.

In this paper we consider the evolution of subgraphs of entire Lipschitz functions, in the case in which either $\varphi$ is regular (see $(2.2)$ ) or $\psi$ is a norm. In particular, we will assume that there exists a Lipschitz continuous function $u_{0}: \mathbb{R}^{N} \rightarrow \mathbb{R}$ such that the initial set $E_{0}$ coincides with $\left\{(x, z) \mid z \leqslant u_{0}(x)\right\}$. By monotonicity of the flow and invariance with respect to translations, the evolution $E_{t}$ is defined for all times and is the subgraph

* The authors were supported by the INDAM-GNAMPA and by the PRIN Project 2019/24 Variational methods for stationary and evolution problems with singularities and interfaces.

Keywords and phrases: Anisotropic mean curvature flow, self-similar solutions, long time behavior.

${ }^{1}$ Department of Statistical Sciences, University of Padova Via Cesare Battisti 141, 35121 Padova, Italy.

2 Universität Duisburg-Essen, Fakultät für Mathematik. Thea-Leymann-Straße 9, 45127, Essen, Germany.

3 Department of Mathematics, University of Pisa, Largo Bruno Pontecorvo 5, 56127 Pisa, Italy.

** Corresponding author: annalisa.cesaroni@unipd.it 
of a Lipschitz function, that is, $E_{t}=\{(x, z) \mid z \leqslant u(x, t)\}$. In Section 2 we describe the main properties of this flow.

Since the evolution is defined for all times, we are interested in the analysis of the long time patterns of the evolution. We recall that evolution of entire Lipschitz graphs in the isotropic setting has been considered in [10], see also [9], whereas the case of fractional mean curvature flow has been considered recently by two of the authors in [5].

As in the isotropic case, the long time attractors of the flow starting from entire Lipschitz graphs are selfsimilar expanding solutions, defined as follows:

Definition 1.1. An expanding homothetic solution is a solution to (1.1) such that $E_{t}=\lambda(t) E_{1}$ where $\lambda(1)=1$ and $\lambda^{\prime}(t) \geqslant 0$ for $t>1$.

Remark 1.2. If $E_{t}$ is an expanding homothetic solution, substituting $E_{t}=\lambda(t) E_{1}$ in (1.1), we get, by scaling properties of the operator $H_{\varphi}$, that

$$
\lambda^{\prime}(t) p \cdot \nu(p)=-\frac{1}{\lambda(t)} \psi(\nu(p)) H_{\varphi}\left(p, E_{1}\right) \quad \forall t>1,
$$

which implies that $\lambda^{\prime}(t) \lambda(t)$ has to be constant. This means that $E_{1}$ is a solution to

$$
c(p \cdot \nu(p))=-\psi(\nu(p)) H_{\varphi}\left(p, E_{1}\right)
$$

for some $c \geqslant 0$. On the other hand, if $E_{1}$ solves (1.2), then the flow defined as $E_{t}:=\sqrt{1+2 c(t-1)} E_{1}$ for $t \geqslant 1$ is an expanding homothetic solution to (1.1).

In Section 3 we characterize the graphical expanding solutions as evolutions issuing from cones, that is, from Lipschitz graphs of positive 1-homogeneous functions, see Theorem 3.1 and Proposition 3.3. Moreover, in Theorem 3.4 we show that Lipschitz graphical evolutions to (1.1) asymptotically approach self-similar expanding solutions, in an appropriate rescaled setting, provided the initial graph is a sublinear perturbations of a cone. More precisely, we introduce the following time rescaling:

$$
\tau(t):=\frac{\log (2 t+1)}{2} \quad \text { and } \quad \tilde{E}_{\tau}:=\frac{1}{\sqrt{2 t+1}} E_{t},
$$

so that the evolution (1.1) of the rescaled flow is governed by the geometric law

$$
\partial_{\tau} \tilde{p} \cdot \tilde{\nu}(\tilde{p})=-\tilde{p} \cdot \tilde{\nu}(\tilde{p})-\psi(\tilde{\nu}(\tilde{p})) H_{\varphi}\left(\tilde{p}, \tilde{E}_{\tau}\right)
$$

and we show that $\tilde{E}_{\tau}$ converge locally in Hausdorff sense as $\tau \rightarrow+\infty$ to a solution to (1.2), with $c=1$.

In the rest of the paper we analyze the long time behavior of the flow (1.1) without rescaling in the case of regular anisotropies, see assumption (2.2) below. In order to rule out possible oscillations in time, it is necessary to assume some decay condition of the initial data at infinity, and in particular we will consider initial graphs which are asymptotically flat or asymptotically approaching mean convex cones.

In Section 4 we consider self-similar expanding solutions starting from Lipschitz mean convex cones. In Theorem 4.3, by constructing appropriate barriers, we show that such solutions are stable with respect to perturbations vanishing at infinity.

In Section 5 we prove that if the initial surface is asymptotically approaching a hyperplane, then the evolution asymptotically flattens out in Hausdorff sense. This result is obtained by comparison with large Wulff shapes, and by constructing appropriate 1-dimensional periodic barriers to the evolution. This approach also provides a different proof for the same result in the isotropic setting, which was obtained by integral estimates on the flow, see $[9,11]$. 


\section{Preliminary Definitions AND RESUlts}

We recall some definitions for anisotropies and related geometric flows (see for instance [3]).

Definition 2.1. Let $\varphi: \mathbb{R}^{N+1} \rightarrow[0,+\infty)$ be a positively 1-homogeneous convex map, such that $\varphi(p)>0$ for all $p \neq 0$. We associate to the surface tension the anisotropy $\varphi^{0}: \mathbb{R}^{N+1} \rightarrow[0+\infty)$ defined as $\varphi^{0}(q):=\sup _{\varphi(p) \leqslant 1} p \cdot q$, which is again convex and positively 1-homogeneous. Observe that if $\varphi \in C^{2}\left(\mathbb{R}^{N+1} \backslash\{0\}\right)$ and $\varphi^{2}$ is uniformly convex, then also $\varphi^{0} \in C^{2}\left(\mathbb{R}^{N+1} \backslash\{0\}\right)$ and $\left(\varphi^{0}\right)^{2}$ is uniformly convex.

When $\varphi$ is regular, the anisotropic mean curvature of a set $E$ at a point $p \in \partial E$ is defined as

$$
H_{\varphi}(p, E)=\operatorname{div}_{\tau}(\nabla \varphi(\nu(p))),
$$

where $\nu(p)$ is the exterior normal vector to $\partial E$ at $p$, and $\operatorname{div}_{\tau}$ is the tangential divergence. In the general case the anisotropic mean curvature can be defined as

$$
H_{\varphi}(p, E)=\operatorname{div}_{\tau}(n(p))
$$

where $n(p)$ is the minimal section of the subdifferential $\partial \varphi(\nu(p))$, that is, the element with minimal norm in the subdifferential, see $[4,15]$.

Definition 2.2 ( $\left.W_{\varphi^{0} \text {-condition}}\right)$. We define the Wulff shape as the convex compact set

$$
W_{\varphi^{0}}:=\left\{q \in \mathbb{R}^{N+1} \mid \varphi^{0}(q) \leqslant 1\right\} .
$$

For any $R>0$ we define the rescaled Wulff shape $R W_{\varphi^{0}}$. It is possible to check that $H_{\varphi}\left(R W_{\varphi^{0}}\right)=\frac{N}{R}$.

We say that $C \subseteq \mathbb{R}^{N+1}$ satisfies the interior (resp. exterior) $R W_{\varphi^{0}}$-condition at $x \in \partial C$ if there exists $y_{x} \in$ $\mathbb{R}^{N+1}$ such that $R W_{\varphi^{0}}+y_{x} \subseteq C$ and $x \in \partial\left(R W_{\varphi^{0}}+y_{x}\right)$ (resp. there exists $y^{x}$ such that $R W_{\varphi^{0}}+y^{x} \subseteq \mathbb{R}^{N+1} \backslash C$ and $\left.x \in \partial\left(R W_{\varphi^{0}}+y^{x}\right)\right)$.

We consider the geometric evolution law (1.1) under the following assumptions on anisotropy and mobility:

$$
\psi: \mathbb{R}^{N+1} \rightarrow[0,+\infty) \text { is continuous, positively 1-homogeneous, and } \psi(p)>0 \quad \forall p \neq 0
$$

and

$$
\begin{gathered}
\text { either } \varphi \in C^{2}\left(\mathbb{R}^{N+1} \backslash\{0\}\right) \text { and } \varphi^{2} \text { is uniformly convex } \\
\text { or } \\
\psi \text { is convex. }
\end{gathered}
$$

Remark 2.3. By positive 1-homogeneity there holds that $\nabla \varphi(\lambda p)=\nabla \varphi(p)$ for every $\lambda>0$ and $p \in \mathbb{R}^{N+1}$ and moreover that $\nabla \varphi(p) \cdot p=\varphi(p)$.

Remark 2.4 (Level set equation). When $\varphi$ is sufficiently regular, that is, (2.2) holds, we associate to the geometric flow (1.1) the following level set equation: Given a uniformly continuous function $U_{0}: \mathbb{R}^{N+1} \rightarrow \mathbb{R}$ such that $E_{0}=\left\{p \in \mathbb{R}^{N+1}: U_{0}(p) \leqslant 0\right\}$ and $\partial E_{0}=\left\{p \in \mathbb{R}^{N+1}: U_{0}(p)=0\right\}$, we consider the following quasi-linear parabolic equation

$$
\left\{\begin{array}{l}
U_{t}-\psi(\nabla U) \operatorname{div}(\nabla \varphi(\nabla U))=0 \\
U(p, 0)=U_{0}(p)
\end{array}\right.
$$

where the solution $U(p, t)$ to $(2.4)$ has to be intended in viscosity sense (see $[2,13])$. When $\varphi$ is not smooth, $\psi$ satisfies (2.3), and the Wulff shape associated to $\psi$ satisfies a uniform interior $R W_{\varphi^{0}}$-condition, it is still possible 
to define a well-posed notion of solution to (2.4) as distributional solution (see [6], Def. 2.5). Finally, in the general case the existence of a unique solution to (2.4) has been obtained in [6] via an approximation argument (for the anisotropies and the mobilities). We recall also that the distributional solution is the locally uniform limit of viscosity solutions to (2.4), when we approximate the anisotropy and the mobility with regular ones, see [7].

We summarize the results about well posedness of the flow (1.1).

Theorem 2.5. There exists a unique continuous solution $U$ to (2.4), to be intended in the sense of [6], that is, the level set flows defined as

$$
\begin{aligned}
& E_{t}^{+}:=\left\{p \in \mathbb{R}^{N+1}: U(p, t) \leqslant 0\right\} \\
& E_{t}^{-}:=\left\{p \in \mathbb{R}^{N+1}: U(p, t)<0\right\}
\end{aligned}
$$

provide a solution (in the appropriate sense) to (1.1).

Moreover, if $U_{0}, V_{0}$ are two uniformly continuous functions such that $U_{0} \leqslant V_{0}$, then $U(p, t) \leqslant V(p, t)$ for all $t>0$ and $p \in \mathbb{R}^{N+1}$.

Finally, if $U_{0}$ is Lipschitz continuous with Lipschitz constant $C$, then

$$
|U(p, t)-U(q, s)| \leqslant C|p-q|+C^{\prime} \sqrt{|t-s|} \quad \forall p, q \in \mathbb{R}^{N+1}, t, s \geqslant 0,
$$

where the constant $C^{\prime}$ depends on $C$. In particular, if there exists a direction $\omega \in \mathbb{R}^{N+1}$ such that $U_{0}(p+\lambda \omega)>$ $U_{0}(p)$ for every $\lambda>0$ and every $p \in \mathbb{R}^{N+1}$, then $U(p+\lambda \omega, t)>U(p, t)$ for every $t>0, \lambda>0, p \in \mathbb{R}^{N+1}$.

Proof. For the existence and uniqueness of solutions to (2.4), and the comparison principle, in the case that (2.2) holds we refer to [2], whereas for the general case in which (2.3) holds we refer to [6], see also Remark 2.4.

The last two properties are a consequence of the comparison principle and the fact that the differential operator is invariant by translations. Indeed, if $U_{0}$ is Lipschitz continuous, and $C$ is the Lipschitz constant of $U_{0}$, then for every fixed $h \in \mathbb{R}^{N+1}$, then $U_{h}(p, t):=U(p+h, t) \pm C|h|$ is a solution to (2.4) with initial datum $U_{0}(p+h) \pm C|h|$. Since $U_{0}(p+h)-C|h| \leqslant U_{0}(p) \leqslant U_{0}(p+h)+C|h|$, by comparison there holds that $U(p+h, t)-C|h| \leqslant U(p, t) \leqslant U(p+h, t)+C|h|$, which implies that

$$
|U(p, t)-U(q, t)| \leqslant C|p-q| \quad \forall p, q \in \mathbb{R}^{N+1}, t>0 .
$$

A similar argument shows that, if $U_{0}(p+\lambda \omega)>U_{0}(p)$ for every $\lambda>0$ then $U(p+\lambda \omega, t)>U(p, t)$.

Finally, observe that if the initial datum is $V_{0}(p)=C|p|$, then by uniqueness, by the fact that $V_{0}(r p)=r V_{0}(p)$ and by the scaling properties of the operator, we get that the solution to (2.4) satisfies $V(p, t)=\frac{1}{r} V\left(r p, r^{2} t\right)$ for every $r>0$ and $t \geqslant 0, p \in \mathbb{R}^{N+1}$. This implies in particular that

$$
V(0, t)=\sqrt{t} V(0,1) \quad \text { for every } t>0 .
$$

To prove the Hölder continuity of $U$, we proceed as follows. We fix $p_{0}$ and observe that $U_{0}\left(p_{0}+p\right) \leqslant$ $C|p|+U_{0}\left(p_{0}\right)$. Hence, by comparison $U\left(p+p_{0}, t\right) \leqslant V(p, t)+U_{0}\left(p_{0}\right)$ and using $(2.5)$, we get that $U\left(p_{0}, t\right) \leqslant$ $\sqrt{t} V(0,1)+U_{0}\left(p_{0}\right)$. Therefore we get that $U\left(p_{0}, t\right)-U\left(p_{0}, 0\right) \leqslant V(0,1) \sqrt{t}$. By translation invariance of the operator, we conclude that for every $s>0$,

$$
U\left(p_{0}, t+s\right)-U\left(p_{0}, s\right) \leqslant V(0,1) \sqrt{t} .
$$

The other inequality is obtained analogously, taking as initial datum $-C|p|$. 
Remark 2.6 (Barriers for the evolution of the Wulff shape). We recall that the evolution of convex sets does not develop fattening, see ([7], Rem. 3.12). Therefore, if $R W_{t}^{ \pm}$is the level set solution to (1.1), with initial datum $R W_{\varphi^{0}}$ as defined in Theorem 2.5, we have that $R W_{t}^{+}=\overline{R W_{t}^{-}}:=R W_{t}$.

Let now $\underline{\psi}:=\min _{\nu \in \mathbb{S}^{N}} \psi(\nu)>0, \bar{\psi}:=\max _{\nu \in \mathbb{S}^{N}} \psi(\nu)>0$, and define $\underline{R}(t):=\sqrt{R^{2}-2 \bar{\psi} N t}$ and $\bar{R}(t):=$ $\sqrt{R^{2}-2 \underline{\psi} N t}$, for $t$ sufficiently small. Then $\underline{R}(t) W_{\varphi^{0}}$ is a subsolution to (1.1) with initial datum $R W_{\varphi^{0}}$, whereas $\bar{R}(t) W_{\varphi^{0}}$ is a supersolution to (1.1) with initial datum $R W_{\varphi^{0}}$. This implies that $\underline{R}(t) W_{\varphi^{0}} \subseteq R W_{t} \subseteq \bar{R}(t) W_{\varphi^{0}}$.

In this paper we consider the case in which the initial datum $E_{0}$ is the subgraph of an entire Lipschitz function. Up to a rotation of coordinates, we may assume that

$$
\exists u_{0}: \mathbb{R}^{N} \rightarrow \mathbb{R}, \text { Lipschitz continuous such that } E_{0}=\left\{(x, z) \in \mathbb{R}^{N+1} \mid z \leqslant u_{0}(x)\right\} .
$$

A direct application of Theorem 2.5 gives the following result on the evolution of Lipschitz graphs.

Corollary 2.7. Assume that $E_{0}$ satisfies (2.6). Then the level set flow satisfies $\bar{E}_{t}^{-}=E_{t}^{+}=\{(x, z) \in$ $\left.\mathbb{R}^{N+1} \mid z \leqslant u(x, t)\right\}$, where $u(x, t)$ is a continuous function such that

$$
|u(x, t)-u(y, s)| \leqslant\left\|\nabla u_{0}\right\|_{\infty}|x-y|+K \sqrt{|t-s|}
$$

for some $K>0$ depending only on the Lipschitz constant $\left\|\nabla u_{0}\right\|_{\infty}$ of $u_{0}$.

When $\varphi$ is regular, that is, (2.2) holds, then $u$ is the viscosity solution to

$$
\left\{\begin{array}{l}
u_{t}+\psi(-\nabla u, 1) \operatorname{div}\left(\nabla_{x} \varphi(-\nabla u, 1)\right)=0 \\
u(x, 0)=u_{0}(x) .
\end{array}\right.
$$

When $\varphi$ is not regular and (2.3) holds, then the solution is intended in the sense of [6], and coincides with the locally uniform limit of viscosity solutions to (2.7) when $\varphi, \psi$ are approximated by regular functions, see [7].

Eventually we recall the following regularity results for solutions to (2.7).

Proposition 2.8. Let $u_{0}$ be a Lipschitz continuous function. Assume that $\varphi$ satisfies (2.2), and that $\nabla^{2} \varphi, \psi$ belong to $C^{0, \beta}\left(\mathbb{R}^{N+1} \backslash\{0\}\right)$ for some $\beta \in(0,1)$.

Then $u(\cdot, t) \in C^{2, \alpha}\left(\mathbb{R}^{N}\right)$ and $u(x, \cdot) \in C^{1, \frac{\alpha}{2}}(0,+\infty)$ for every $(x, t) \in \mathbb{R}^{N} \times(0,+\infty)$ and for some $\alpha \in(0,1)$. Moreover for every $t_{0}>0$ there exists a positive constant $C$, depending on $t_{0}, \varphi, \psi$ and the Lipschitz constant of $u_{0}$, such that

$$
\|\nabla u(\cdot, t)\|_{C^{1, \alpha}\left(\mathbb{R}^{N}\right)}+\left\|u_{t}(x, \cdot)\right\|_{C^{0, \frac{\alpha}{2}}\left(t_{0},+\infty\right)} \leqslant C
$$

for every $(x, t) \in \mathbb{R}^{N} \times\left(t_{0},+\infty\right)$.

Proof. If the initial datum $u_{0}$ belongs to $C^{2, \alpha}\left(\mathbb{R}^{N}\right)$, then by Section 6 of [1] (see also [17, 18]) there exists a solution $u: \mathbb{R}^{N} \times(0, T) \rightarrow \mathbb{R}$ for some $T>0$ such that $u(\cdot, t) \in C^{2, \alpha}\left(\mathbb{R}^{N}\right)$ and $u(x, \cdot) \in C^{1, \frac{\alpha}{2}}(0, T)$ for every $(x, t) \in \mathbb{R}^{N} \times(0, T)$. Then, by standard results for quasilinear parabolic equations with Hölder continuous coefficients, see e.g. Proposition 9.5, Proposition 9.6of [1], we have that the norm of the solution $u$ depends only on $\psi, \varphi$ and the Lipschitz constant of $u_{0}$. It follows that $T=+\infty$ and (2.8) holds.

If $u_{0}$ is only Lipschitz continuous we approximate $u_{0}$ with initial data in $C^{2, \alpha}\left(\mathbb{R}^{N}\right)$, and then conclude by stability of solutions to (2.7) with respect to local uniform convergence. 
Proposition 2.9. Let $u_{0} \in C^{1,1}\left(\mathbb{R}^{N}\right)$ with $\left\|u_{0}\right\|_{C^{1,1}\left(\mathbb{R}^{N}\right)} \leqslant C$ and let (2.2) hold. Then the viscosity solution $u$ of (2.7) satisfies

$$
|u(x, t)-u(y, s)| \leqslant\left\|\nabla u_{0}\right\|_{\infty}|x-y|+K|t-s|
$$

for some $K>0$ depending only on $\left\|u_{0}\right\|_{C^{1,1}\left(\mathbb{R}^{N}\right)}$ and on $\psi$ and $\varphi$. Moreover $u(\cdot, t)$ is uniformly of class $C^{1, \alpha}\left(\mathbb{R}^{N}\right)$, for any fixed $t>0$ and for all $\alpha \in(0,1)$, with $C^{1, \alpha}$ norm bounded independently of $t>0$.

Proof. First of all let $u(x, t)$ be the viscosity solution to (2.7). By Corollary 2.7, it is Lipschitz continuous with $\|\nabla u(x, t)\|_{\infty} \leqslant\left\|\nabla u_{0}\right\|_{\infty}$. Let $C^{\prime}:=\left\|\psi\left(-\nabla u_{0}, 1\right) \operatorname{div}\left(\nabla \varphi\left(-\nabla u_{0}, 1\right)\right)\right\|_{\infty}$. Note that $u_{0}(x) \pm C^{\prime} t$ are respectively a supersolution and a subsolution to (2.7), so that by comparison principle, see Theorem 2.5, we get

$$
u_{0}(x)-C^{\prime} t \leqslant u(x, t) \leqslant u_{0}(x)+C^{\prime} t \quad \text { for all } t \geqslant 0 .
$$

Let us fix $\tau>0$. Observe that the functions $v^{ \pm}(x, t):=u(x, t) \pm \sup _{x}\left|u(x, \tau)-u_{0}(x)\right|$ are respectively a supersolution and a subsolution to $(2.7)$ with $v^{-}(x, 0) \leqslant u(x, \tau) \leqslant v^{+}(x, 0)$, whence by comparison $v^{-}(x, t) \leqslant$ $u(x, t+\tau) \leqslant v^{+}(x, t)$, which gives

$$
|u(x, t+\tau)-u(x, t)| \leqslant \sup _{x}\left|u(x, \tau)-u_{0}(x)\right| \leqslant C^{\prime} \tau .
$$

This implies that $u(x, \cdot)$ is Lipschitz continuous with $\left|u_{t}(x, t)\right| \leqslant C^{\prime}$. This in turn gives, recalling that $\nabla u$ is bounded, that

$$
-C \leqslant \operatorname{div}(\nabla \varphi(-\nabla u, 1)) \leqslant C \quad \text { for all } x \in \mathbb{R}^{N} \text { and } t>0
$$

By elliptic regularity theory for viscosity solutions (see [20]), this implies that for every $t>0, u(\cdot, t) \in C^{1, \alpha}\left(\mathbb{R}^{N}\right)$ for every $\alpha<1$.

\section{Self-Similar EXPANDing SOlutions AND CONVERGENCE OF THE RESCALED FLOW}

We discuss the properties of solutions to (1.1) starting from Lipschitz cones, that is, subgraphs of Lipschitz continuous and positively 1-homogeneous functions $\bar{u}$ :

$$
\exists C>0 \quad|\bar{u}(x)-\bar{u}(y)| \leqslant C|x-y| \quad \bar{u}(r x)=r \bar{u}(x) \quad \forall r>0, x, y \in \mathbb{R}^{N} .
$$

Then we consider the long time behavior of solutions starting from sublinear perturbations of Lipschitz cones.

Theorem 3.1. Assume (2.1) and that either (2.2) holds, or (2.3) holds. Let $\bar{u}: \mathbb{R}^{N} \rightarrow \mathbb{R}$ be as in (3.1) and let $\bar{E}_{0}$ be the subgraph of $\bar{u}$. Then, for every $t>0$, the evolution $\bar{E}_{t}$ of (1.1) with initial datum $\bar{E}_{0}$ satisfies for $p \in \partial \bar{E}_{t}$

$$
p \cdot \nu=-2 t \psi(\nu(p)) H_{\varphi}\left(p, \bar{E}_{t}\right)
$$

that is, the flow starting from $\bar{E}_{0}$ is an expanding homothetic solution to (1.1). Writing $\bar{E}_{t}$ as subgraph of a function $\bar{u}(\cdot, t)$ we have that for all $T>0$

$$
\lim _{t \rightarrow \infty} \bar{u}(x, t+T)-\bar{u}(x, t)=0 \quad \text { locally uniformly in } \mathbb{R}^{N} .
$$


Finally, if either $\bar{u} \in C^{1}\left(\mathbb{R}^{N} \backslash\{0\}\right)$ or $\bar{E}_{0}$ satisfies the exterior and interior $R_{x} W_{\varphi^{0}}$-condition at every $x \in$ $\partial \bar{E}_{0} \backslash\{0\}$ (see Def. 2.2) with $R_{x}$ possibly depending on $x$, then

$$
\lim _{|x| \rightarrow+\infty} \bar{u}(x, t)-\bar{u}(x)=0 \quad \text { for every } t>0
$$

Proof. We define, for $x \in \mathbb{R}^{N}$ and $z \in \mathbb{R}, \bar{U}(x, z)=z-\bar{u}(x)$. So, by (3.1) we get that for every $r>0$, there holds that $\bar{U}(r x, r z)=r \bar{U}(x, z)$. Arguing as in Theorem 2.5, by uniqueness of solutions to (2.4) and by the rescaling properties of the operator, we get that $\bar{U}(x, z, t)=\frac{1}{r} \bar{U}\left(r x, r z, r^{2} t\right)$ for all $r>0$, where $\bar{U}(x, z, t)$ is the unique solution to (2.4) with initial datum $\bar{U}(x, z)$. Therefore recalling the definition of the level set flow, we get that $(x, z) \in \bar{E}_{t}$ if and only if $(r x, r z) \in \bar{E}_{r^{2} t}$, which in turns gives that $\bar{E}_{t}=r \bar{E}_{t / r^{2}}$ for all $t, r>0$. The previous rescaling identity reads

$$
\bar{u}(x, t)=\frac{1}{r} \bar{u}\left(r x, r^{2} t\right), \quad r, t>0 \quad x \in \mathbb{R}^{N} .
$$

Letting $r:=t^{-\frac{1}{2}}$ for $t>0$, we get

$$
\bar{u}(x, t)=t^{\frac{1}{2}} \bar{u}\left(x t^{-\frac{1}{2}}, 1\right) .
$$

This implies that, if $p \in \partial \bar{E}_{1}$ then $p t^{\frac{1}{2}} \in \partial \bar{E}_{t}$ and

$$
H_{\varphi}\left(p t^{\frac{1}{2}}, \bar{E}_{t}\right)=t^{-\frac{1}{2}} H_{\varphi}\left(p, \bar{E}_{1}\right)
$$

Substituting in (1.1) we get that $\bar{E}_{1}$ solves (1.2) with $c^{-1}=2$. The same argument holds substituting $t=1$ with another time $t>0$.

We observe that, by rescaling property $(3.3)$, and by the fact that $\bar{u}(\cdot, t)$ is Lipschitz continuous with the same Lipschitz constant as $\bar{u}$, for every $T>0$ and $t>0$ we have

$$
\begin{aligned}
|\bar{u}(x, t+T)-\bar{u}(x, t)|= & \left|(t+T)^{\frac{1}{2}} \bar{u}\left(x(t+T)^{-\frac{1}{2}}, 1\right)-t^{\frac{1}{2}} \bar{u}\left(x t^{-\frac{1}{2}}, 1\right)\right| \\
\leqslant & (t+T)^{\frac{1}{2}}\left|\bar{u}\left(x(t+T)^{-\frac{1}{2}}, 1\right)-\bar{u}\left(x t^{-\frac{1}{2}}, 1\right)\right| \\
& +\left|(t+T)^{\frac{1}{2}}-t^{\frac{1}{2}}\right|\left|\bar{u}\left(x t^{-\frac{1}{2}}, 1\right)\right| \\
\leqslant & C(t+T)^{\frac{1}{2}}|x|\left|(t+T)^{-\frac{1}{2}}-t^{-\frac{1}{2}}\right|+C\left|(t+T)^{\frac{1}{2}}-t^{\frac{1}{2}}\right||x| t^{-\frac{1}{2}} \\
\leqslant & C|x|\left(\left(1+\frac{T}{t}\right)^{\frac{1}{2}}-\left(1-\frac{T}{t}\right)^{\frac{1}{2}}\right) \leqslant \frac{C T|x|}{t} .
\end{aligned}
$$

Sending $t \rightarrow+\infty$, we get the result.

We now show that, if $\bar{E}_{0}$ satisfies the exterior and interior $W_{\varphi^{0}}$-condition, the expanding solution is asymptotic at infinity to the initial cone, by comparison with the shrinking Wulff shapes constructed in Remark 2.6.

Note that by positive 1-homogeneity of the function $\bar{u}$, for every $R>0$, there exists $K>0$ such that $\bar{E}_{0}$ satisfies the exterior and interior $R W_{\varphi^{0}}$-condition at every $(x, \bar{u}(x)) \in \partial \bar{E}_{0}$, with $|x| \geqslant K$. Therefore we get

$$
\bigcup_{|x| \geqslant K}\left(R W_{\varphi_{0}}+y_{x}\right) \subseteq \bar{E}_{0} \subseteq \overline{\mathbb{R}^{N+1} \backslash \bigcup_{|x| \geqslant K}\left(R W_{\varphi_{0}}+y^{x}\right)},
$$


where $y_{x}, y^{x}$ are introduced in Definition 2.2. By comparison (see Rem. 2.6 and Thm. 2.5) we have that

$$
\bigcup_{|x| \geqslant K}\left(\underline{R}(t) W_{\varphi_{0}}+y_{x}\right) \subseteq \bar{E}_{t} \subseteq \overline{\mathbb{R}^{N+1} \backslash \bigcup_{|x| \geqslant K}\left(\underline{R}(t) W_{\varphi_{0}}+y^{x}\right)}
$$

where $\underline{R}(t):=\sqrt{R^{2}-2 N \bar{\psi} t}$ is defined in Remark 2.6. From the previous inclusions, we deduce that, for all $|x| \geqslant K$ and $t<\frac{R^{2}}{2 N \bar{\psi}}$,

$$
|\bar{u}(x, t)-\bar{u}(x)| \leqslant C(R-\underline{R}(t)) \leqslant C \frac{N \bar{\psi} t}{R}
$$

where $C$ is a constant which depends on $\varphi$ and on the Lipschitz constant of $\bar{u}$. This implies the conclusion, sending $R \rightarrow+\infty$.

A similar argument can be used to prove the same result when $\bar{u} \in C^{1}\left(\mathbb{R}^{N} \backslash\{0\}\right)$. For it we define $v_{\lambda}(x):=$ $\bar{u}(\lambda e+x)-\bar{u}(\lambda e)$ where $\lambda>0$ and $e \in \mathbb{R}^{N}$ with $|e|=1$. For every compact set $K$, since $\nabla \bar{u}$ is continuous in $\mathbb{R}^{N} \backslash\{0\}$, we get that as $\lambda \rightarrow+\infty$

$$
\nabla v_{\lambda}(x)=\nabla \bar{u}(\lambda e+x)=\nabla \bar{u}\left(e+\frac{x}{\lambda}\right) \rightarrow \nabla \bar{u}(e)
$$

uniformly in $x$ and $e$. Hence, letting $f_{e}(x):=\nabla \bar{u}(e) \cdot x$ we conclude that

$$
\lim _{\lambda \rightarrow \infty}\left\|v_{\lambda}-f_{e}\right\|_{C^{1}(K)}=0
$$

uniformly in $e$, with $|e|=1$.

This implies that there are functions $\alpha(r), \varepsilon(r)>0, r>0$, with $\alpha(r) \rightarrow \infty$ and $\varepsilon(r) \rightarrow 0$ as $r \rightarrow \infty$ so that for large $|x|$, there exist $y_{x}, y^{x} \in \mathbb{R}^{N+1}$ such that

$$
d\left((x, \bar{u}(x)), \alpha(|x|) W_{\varphi_{0}}+y_{x}\right), d\left((x, \bar{u}(x)), \alpha(|x|) W_{\varphi_{0}}+y^{x}\right) \leqslant \varepsilon(|x|),
$$

and eventually

$$
\left(\alpha(|x|) W_{\varphi_{0}}+y_{x}\right) \subseteq \bar{E}_{0} \subseteq \overline{\mathbb{R}^{N+1} \backslash\left(\alpha(|x|) W_{\varphi_{0}}+y^{x}\right)}
$$

So, the thesis follows from the same argument as above.

Remark 3.2. If we assume that $\nabla^{2} \varphi, \psi$ belong to $C^{0, \beta}\left(\mathbb{R}^{N+1} \backslash\{0\}\right)$ for some $\beta \in(0,1)$, then there exists $C>0$ such that

$$
\sup _{x \in \mathbb{R}^{N}}\left|\operatorname{div}\left(\nabla_{x} \varphi(-\nabla \bar{u}(x, t), 1)\right)\right| \leqslant C t^{-\frac{1}{2}} \quad \forall t>0 .
$$

Indeed due to (3.4) it is sufficient to check that there exists $C>0$ such that

$$
\sup _{x \in \mathbb{R}^{N}}\left|\operatorname{div}\left(\nabla_{x} \varphi(-\nabla \bar{u}(x, 1), 1)\right)\right| \leqslant C,
$$

and this is a consequence of Proposition 2.8. 
Moreover, if (3.5) is satisfied, then $\left|\bar{u}_{t}(x, t)\right| \leqslant C^{\prime} t^{-\frac{1}{2}}$, for some $C^{\prime}>0$ depending on $C$ and on the Lipschitz norm of $u_{0}$. Integrating in $t$ we get

$$
|\bar{u}(x, t+T)-\bar{u}(x, t)| \leqslant C^{\prime}(\sqrt{t+T}-\sqrt{t}) \quad \text { for all } T>0
$$

hence, for every $T>0$ we conclude that

$$
\lim _{t \rightarrow+\infty}|\bar{u}(x, t+T)-\bar{u}(x, t)|=0 \quad \text { uniformly in } \mathbb{R}^{N}
$$

On the other hand, every homothetically expanding solution $E_{t}$ to (1.1) which is the subgraph of a Lipschitz continuous function has a backward in time extension which starts from a subgraph of a suitable Lipschitz and 1-homogeneous function.

Proposition 3.3. Assume that $E_{1}$ is a solution to (1.2) for some $c>0$, such that there exists a Lipschitz continuous function $u_{1}: \mathbb{R}^{N} \rightarrow \mathbb{R}$ for which $E_{1}=\left\{(x, z) \mid z \leqslant u_{1}(x)\right\}$. Let $u(x, t)$ be the solution to (2.7) in $\mathbb{R}^{N} \times(1,+\infty)$ with initial datum $u(x, 1)=u_{1}(x)$. Then we may extend continuously $u(x, t)$ in $\mathbb{R}^{N} \times\left(t_{0},+\infty\right)$, where $t_{0}=1-\frac{1}{2 c}$, and moreover it holds

$$
\lim _{t \rightarrow t_{0}^{+}} u(x, t)=\bar{u}(x) \quad \text { locally uniformly }
$$

where $\bar{u}: \mathbb{R}^{N} \rightarrow \mathbb{R}$ is a positively 1-homogeneous Lipschitz function.

Proof. Since $E_{1}$ solves (1.2), we have that

$$
E_{t}=\lambda(t) E_{1}, \quad \text { where } \lambda(t):=(2 c(t-1)+1)^{\frac{1}{2}}, \quad t \geq 1
$$

Therefore the solution $u$ to $(2.7)$ in $\mathbb{R}^{N} \times(1,+\infty)$ with initial datum $u(x, 1)=u_{1}(x)$ is given by

$$
u(x, t)=\lambda(t) u_{1}\left(\frac{x}{\lambda(t)}\right), \quad t>1, x \in \mathbb{R}^{N}
$$

By definition $\lambda(t)$ is well defined for every $t>t_{0}=1-\frac{1}{2 c}$, and so $u$ can be extended to a continuous function in $\mathbb{R}^{N} \times\left(t_{0},+\infty\right)$, which is Lipschitz continuous in $x$, with the same Lipschitz constant as $u_{1}$. We compute now the limit as $t \rightarrow t_{0}^{+}$, that is the limit as $\lambda(t) \rightarrow 0^{+}$in (3.6). For $r \in(0,1)$, we define

$$
v_{r}(x):=r u_{1}\left(\frac{x}{r}\right)
$$

Note that $\left(v_{r}\right)_{r}$ are equi-Lipschitz and locally bounded so that, up to subsequences, there exists the locally uniform limit of $v_{r}$ as $r \rightarrow 0^{+}$by Arzelà-Ascoli Theorem.

In order to conclude, we need to show that such limit is unique, i.e., it does not depend on the subsequence. If this is true, it is easy to check that $\bar{u}(x):=\lim _{r \rightarrow 0^{+}} r u_{1}\left(\frac{x}{r}\right)$ satisfies $(3.1)$. To prove the whole convergence, we observe that by (3.6)

$$
v_{r}(x)=u\left(x, \lambda^{-1}(r)\right) \quad \forall r>0
$$

Let $r_{n} \rightarrow 0^{+}$so that $\lim _{n \rightarrow \infty} v_{r_{n}}(x)=: \bar{v}(x)$ locally uniformly. We set

$$
u_{n}(x, t):=u\left(x, t+\lambda^{-1}\left(r_{n}\right)\right)
$$


Then $u_{n}$ is the solution to (2.7) in $\mathbb{R}^{N} \times(0,+\infty)$ with initial datum $u_{n}(x, 0)=u\left(x, \lambda^{-1}\left(r_{n}\right)\right)=v_{r_{n}}(x)$. Recalling that $v_{r_{n}}(x) \rightarrow \bar{v}(x)$ locally uniformly, and that $v_{r_{n}}$ and $\bar{v}$ are equi-Lipschitz functions, by Corollary 2.7, we get that, up to subsequences, $u_{n}(x, t) \rightarrow \bar{v}(x, t)$ locally uniformly in $(x, t)$, for some function $\bar{v}(x, t)$. By stability of solutions to (2.7) with respect to uniform convergence, we get that $\bar{v}(x, t)$ is the solution to (2.7) in $R^{N} \times(0,+\infty)$ with initial datum $\bar{v}(x, 0)=\bar{v}(x)$. Now observe that by definition $u_{n}(x, t)=u\left(x, t+\lambda^{-1}\left(r_{n}\right)\right) \rightarrow u\left(x, t+t_{0}\right)$ for every $t>0$, therefore $\bar{v}(x, t)=u\left(x, t+t_{0}\right)$ for every $t>0$. This implies that the limit $\bar{v}$ is independent of the subsequence $r_{n}$.

We now provide the locally uniform convergence to self-similar expanding solutions, in the rescaled setting (1.3), if the initial Lipschitz graph is a sublinear perturbation of a cone. A similar result has been obtained in [10] for the isotropic case, and in [5] for the fractional mean curvature flow. Note that, if the flow $E_{t}$ is the subgraph of the solution $u(x, t)$ to $(2.7)$, then the rescaled flow $\tilde{E}_{\tau}$ defined in (1.3) is the subgraph of the rescaled function

$$
\tilde{u}(y, \tau):=e^{-\tau} u\left(y e^{\tau}, \frac{e^{2 \tau}-1}{2}\right)
$$

Theorem 3.4. Let $u_{0}$ be a Lipschitz continuous function, such that there exist $\bar{u}$ which satisfies (3.1), and constants $K>0, \delta \in(0,1)$ for which there holds

$$
\left|u_{0}(x)-\bar{u}(x)\right| \leqslant K(1+|x|)^{1-\delta} .
$$

Let $\tilde{u}(y, \tau)$ be the rescaled function as defined in (3.7), where $u$ is the solution to (2.7) with initial data $u_{0}$ and let $\bar{u}(x, t)$ be the solution to (2.7) with initial datum $\bar{u}$. Then

$$
\lim _{\tau \rightarrow+\infty} \tilde{u}(y, \tau)=\bar{u}\left(y, \frac{1}{2}\right) \quad \text { locally uniformly. }
$$

Proof. We argue as in the proof of ([5], Thm. 5.1). Let $\chi:(0,+\infty) \rightarrow(0,+\infty)$ be a smooth function such that $\chi(k) \equiv 0$ if $k<1$ and $\chi(k) \equiv 1$ if $k>2$. Define for $r>1$

$$
u_{0}^{r}(x)=\bar{u}(x)+\chi\left(\frac{|x|}{r}\right)\left(u_{0}(x)-\bar{u}(x)\right) .
$$

Then our assumption implies that

$$
\left|u_{0}(x)-u_{0}^{r}(x)\right| \leqslant K(1+2 r)^{1-\delta} \quad \forall x \in \mathbb{R}^{N} .
$$

By the comparison principle we deduce that

$$
u_{r}(x, t)-K(1+2 r)^{1-\delta} \leqslant u(x, t) \leqslant u_{r}(x, t)+K(1+2 r)^{1-\delta} \quad \forall x \in \mathbb{R}^{N}, t>0
$$

where $u, u_{r}$ are the solutions to (2.7) respectively with initial data $u_{0}$ and $u_{0}^{r}$. Then passing to the rescaled functions $\tilde{u}$ and $\tilde{u}^{r}$ as defined in (3.7), we obtain

$$
\tilde{u}^{r}(y, \tau)-K(1+2 r)^{1-\delta} e^{-\tau} \leqslant \tilde{u}(y, \tau) \leqslant \tilde{u}^{r}(y, \tau)+K(1+2 r)^{1-\delta} e^{-\tau} \quad \forall y \in \mathbb{R}^{N}, \tau>0 .
$$

On the other hand,

$$
\bar{u}(x)-\frac{2 K}{r^{\delta}}|x| \leqslant u_{0}^{r}(x) \leqslant \bar{u}(x)+\frac{2 K}{r^{\delta}}|x| .
$$


Let $\bar{u}_{ \pm r}$ be the solution to (2.7) with initial datum respectively $\bar{u}(x) \pm \frac{2 K}{r^{\delta}}|x|$. By the comparison principle

$$
\bar{u}_{-r}(x, t) \leqslant u^{r}(x, t) \leqslant \bar{u}_{+r}(x, t) \quad \forall x \in \mathbb{R}^{N}, t>0 .
$$

Note that $\bar{u}(x) \pm \frac{2 K}{r^{\delta}}|x|$ satisfy (3.1) with Lipschitz constant $\|D \bar{u}\|_{\infty}+\frac{2 K}{r^{\delta}} \leqslant\|D \bar{u}\|_{\infty}+2 K$. By the rescaling properties of $\bar{u}_{ \pm r}$, see (3.3), we get that when we apply the rescaling (3.7) to $\bar{u}_{ \pm r}$ we obtain $\bar{u}_{ \pm r}\left(y, \frac{1-e^{-2 \tau}}{2}\right)$. Then, passing in (3.9) to the rescaled functions as defined in (3.7) we get

$$
\bar{u}_{-r}\left(y, \frac{1-e^{-2 \tau}}{2}\right) \leqslant \tilde{u}^{r}(y, \tau) \leqslant \bar{u}_{+r}\left(y, \frac{1-e^{-2 \tau}}{2}\right) .
$$

Recalling that $\bar{u}(x) \pm \frac{2 K}{r^{\delta}}|x|$ are Lipschitz functions with Lipschitz constant bounded by $\|D \bar{u}\|_{\infty}+2 K$, by Corollary 2.7 we get that there exists $B$ depending only on $\|D \bar{u}\|_{\infty}$ and $K$ such that

$$
\bar{u}_{-r}\left(y, \frac{1}{2}\right)-B e^{-\tau} \leqslant \tilde{u}^{r}(y, \tau) \leqslant \bar{u}_{+r}\left(y, \frac{1}{2}\right)+B e^{-\tau} .
$$

Therefore by (3.8) we conclude that

$$
\bar{u}_{-r}\left(y, \frac{1}{2}\right)-\left(B+K(1+2 r)^{1-\delta}\right) e^{-\tau} \leqslant \tilde{u}(y, \tau) \leqslant \bar{u}_{+r}\left(y, \frac{1}{2}\right)+\left(B+K(1+2 r)^{1-\delta}\right) e^{-\tau},
$$

for all $y \in \mathbb{R}^{N}, \tau>0$ and $r>1$.

Notice that $\bar{u}_{ \pm r}\left(y, \frac{1}{2}\right) \rightarrow \bar{u}\left(y, \frac{1}{2}\right)$ as $r \rightarrow+\infty$, locally uniformly in $y$ by stability of solutions with respect to local uniform convergence, since $\bar{u}(x) \pm \frac{2 K}{r^{\delta}}|x| \rightarrow \bar{u}(x)$ locally uniformly. Therefore, taking $r=e^{\tau}$ in the previous inequality and letting $\tau \rightarrow+\infty$, we obtain the local uniform convergence of $\tilde{u}$.

Remark 3.5. If $u_{0}-\bar{u} \in L^{\infty}\left(\mathbb{R}^{N}\right)$, the convergence result in Theorem 3.4 can be strengthened to uniform convergence. In the isotropic case, the uniform convergence has been obtained in [10] under the assumptions of Theorem 3.4, by using maximum principle and integral estimates.

\section{Stability of SELF-Similar SOLUTions ASYMPtotic TO MEAN CONVEX CONES}

In this section we assume that the anisotropy is regular, that is, (2.2) holds, and we address the issue of the stability with respect to perturbations vanishing at infinity in the case of mean convex cones. The same problem has been considered in the isotropic setting in [9].

So we consider self-similar expanding solutions starting from initial data which satisfy the following condition: $\bar{u}$ is as in (3.1) and moreover

$$
\bar{u} \in C^{2}\left(\mathbb{R}^{N} \backslash\{0\}\right), \text { and } \operatorname{div}\left(\nabla_{x} \varphi(-\nabla \bar{u}, 1)\right)<0 \text { in the viscosity sense. }
$$

Note that the assumption in (4.1) implies that the epigraph of $\bar{u}$, that is, the set $\{(x, z) \mid z \geqslant \bar{u}(x)\}$ is a mean convex set.

We first show that mean convexity is preserved and that the homothetic solution $\bar{u}(x, t)$ always lies above $\bar{u}$.

Lemma 4.1. Let $\bar{u}$ be as in (3.1) and (4.1) and let $\bar{u}(x, t)$ be the solution to (2.7) with initial datum $\bar{u}$. Then $\bar{u}(x, t+s) \geqslant \bar{u}(x, t)$ for every $t \geqslant 0$ and $s>0$, and moreover $\bar{u}(0, t)=\sqrt{t} \bar{u}(0,1)>0$ for $t>0$. 
Proof. Condition (4.1) implies that $\bar{u}(x)$ is a stationary subsolution to (2.7), so by comparison $\bar{u}(x, t) \geqslant \bar{u}(x)$. Again by comparison and the semigroup property we get that for all $s>0$, there holds $\bar{u}(x, t+s) \geqslant \bar{u}(x, t)$.

In particular we get that $\bar{u}(0, t) \geqslant \bar{u}(0)=0$, moreover by $(3.3), \bar{u}(0, t)=\sqrt{t} \bar{u}(0,1)$. Observe that since $\bar{u}(0,1)$ is a solution to $(1.2)$ with $c=1 / 2$, then $\operatorname{div}\left(\nabla_{x} \varphi(-\nabla \bar{u}, 1)\right) \in L_{l o c}^{\infty}$ and by elliptic regularity theory [20], recalling (2.2), this implies that $\bar{u}(\cdot, 1) \in C^{1, \alpha}\left(\mathbb{R}^{N}\right)$ for every $\alpha<1$. It follows that $\bar{u}(0,1)>0$.

Proposition 4.2. Let $\bar{u}$ be as in (3.1) and (4.1) and let $u_{0}: \mathbb{R}^{N} \rightarrow \mathbb{R}$ be a Lipschitz continuous function such that

$$
\lim _{|x| \rightarrow+\infty} u_{0}(x)-\bar{u}(x)=0 .
$$

Then for every $\delta>0$ there exists $t_{\delta}>0$ such that

$$
u(x, t) \geqslant \bar{u}(x)-\delta, \quad t \geqslant t_{\delta}
$$

where $u(x, t)$ is the viscosity solution to (2.7) with initial datum $u_{0}$.

Proof. The proof is based on the construction of a barrier for the evolution. First of all observe that by assumption, there exists $m>0$ such that $u_{0}(x) \geqslant \bar{u}(x)-m$ for all $x \in \mathbb{R}^{N}$. Moreover, for every $\delta>0$ there exists $R(\delta)>0$ such that $u_{0}(x) \geqslant \bar{u}(x)-\delta$ for every $|x| \geqslant R(\delta)$.

Let $\chi:[0,+\infty) \rightarrow[0,1]$ be a smooth function such that $\chi(s)=1$ if $s<1, \chi(s)=0$ if $s>2$. Then for every $r>0$, we define $b_{r}(x):=\bar{u}(x)-r \chi(|x|)$. Then $b_{r} \in C^{2}\left(\mathbb{R}^{N} \backslash\{0\}\right)$, moreover $b_{r}(x) \leqslant \bar{u}(x)$ for all $x$, in particular $b_{r}(x)=\bar{u}(x)$ if $|x|>2$ and $b_{r}(x)=\bar{u}(x)-r$ if $|x| \leqslant 1$. Finally we get

$$
\lim _{r \rightarrow 0} \max _{|x| \in[1,2]} \operatorname{div}\left(\nabla_{x} \varphi\left(-\nabla b_{r}(x), 1\right)\right) \leqslant \max _{|x| \in[1,2]} \operatorname{div}\left(\nabla_{x} \varphi(-\nabla \bar{u}, 1)\right)=-c<0 .
$$

Choosing $r$ sufficiently small, we get that $\operatorname{div}\left(\nabla_{x} \varphi\left(-\nabla b_{r}(x), 1\right)\right)<0$ in the viscosity sense for all $x$. Now, let us fix $r_{0}$, and $b_{r_{0}}$ the corresponding function such that the previous condition is satisfied. In particular $b_{r_{0}}$ is a stationary subsolution to $(2.7)$.

We claim that there exists $\lambda>0$ such that

$$
u(x, t) \geqslant w(x, t):=\sup \left(\bar{u}(x, t)-m, \lambda b_{r_{0}}\left(\frac{x}{\lambda}\right)-\delta\right)
$$

where $\bar{u}(x, t)$ is the solution to (2.7) with initial datum $\bar{u}$. Note that the function $w(x, t)$ is a subsolution to (2.7), since it is the supremum between two subsolutions. Therefore to check the claim, it is sufficient to show that $u(x, 0)=u_{0}(x) \geqslant w(x, 0)=\sup \left(\bar{u}(x)-m, \lambda b_{r_{0}}\left(\frac{x}{\lambda}\right)-\delta\right)$. First of all, by assumption we know that $u_{0}(x) \geqslant \bar{u}(x)-m$ for all $x$. On the other hand, observe that if $\lambda>\max \left(R(\delta), m / r_{0}\right)$, by definition of $\chi$ and the positive 1-homogeneity of $\bar{u}$, there holds that for $|x| \leqslant R(\delta)<\lambda$,

$$
\lambda b_{r_{0}}\left(\frac{x}{\lambda}\right)=\bar{u}(x)-\lambda r_{0} \chi\left(\frac{|x|}{\lambda}\right)=\bar{u}(x)-\lambda r_{0} \leqslant \bar{u}(x)-m \leqslant u_{0}(x) .
$$

On the other hand if $|x| \geqslant R(\delta)$, by assumption and our construction of $b_{r}, u_{0}(x) \geqslant \bar{u}(x)-\delta \geqslant \lambda b_{r_{0}}\left(\frac{x}{\lambda}\right)-\delta$.

Therefore, by comparison, (4.2) holds for every $\lambda>\max \left(R(\delta), m / r_{0}\right)$. We fix $\lambda_{0}$ which satisfies this condition.

Now, observe that by Lipschitz continuity and Lemma 4.1, there holds $\bar{u}(x, t)-m \geqslant \sqrt{t} \bar{u}(0,1)-\|\nabla \bar{u}\|_{\infty}|x|-$ $m$. Therefore there exists $t_{\delta}$ such that $\bar{u}(x, t)-m \geqslant \bar{u}(x)$, for all $|x| \leqslant 2 \lambda_{0}$ and for all $t \geqslant t_{\delta}$ and then, in turn, by (4.2), we get that $u(x, t) \geqslant \bar{u}(x)$ for all $t \geqslant t_{\delta}$ and $|x| \leqslant 2 \lambda_{0}$. On the other hand, if $|x|>2 \lambda_{0}$ there holds by definition that $\lambda_{0} b_{r_{0}}\left(\frac{x}{\lambda_{0}}\right)-\delta=\bar{u}(x)-\delta$ and then again by (4.2), $u(x, t) \geqslant \bar{u}(x)-\delta$ for all $t$, for all $|x|>2 \lambda_{0}$. So, we get the conclusion. 
We conclude with the following stability result.

Theorem 4.3. Assume that (2.2) holds. Let $u_{0}: \mathbb{R}^{N} \rightarrow \mathbb{R}$ be a Lipschitz continuous function and $\bar{u}$ satisfies (3.1), and (4.1) such that

$$
\lim _{|x| \rightarrow+\infty} u_{0}(x)-\bar{u}(x)=0 .
$$

Then,

$$
\lim _{t \rightarrow+\infty} u(x, t)-\bar{u}(x, t)=0 \quad \text { locally uniformly in } \mathbb{R}^{N}
$$

where $u(x, t), \bar{u}(x, t)$ are the solutions to (2.7) with initial datum $u_{0}, \bar{u}$. If moreover $\nabla^{2} \varphi, \psi$ belong to $C^{0, \beta}\left(\mathbb{R}^{N+1} \backslash\right.$ $\{0\})$ for some $\beta \in(0,1)$, then the convergence is uniform in $\mathbb{R}^{N}$.

Proof. First of all we observe that by Proposition 4.2 and the comparison principle, there holds that for all $\delta>0$ there exists $t_{\delta}$ such that for all $t>t_{\delta}$,

$$
u(x, t) \geqslant \bar{u}\left(x, t-t_{\delta}\right)-\delta .
$$

We fix now $\varepsilon>0$ and $R_{\varepsilon}>0$ such that $u_{0}(x) \leqslant \bar{u}(x)+\varepsilon$ for all $|x|>R_{\varepsilon}$. Therefore, by Lemma 4.1, we get that for all $t>0$,

$$
u_{0}(x) \leqslant \bar{u}(x)+\varepsilon \leqslant \bar{u}(x, t)+\varepsilon \quad \text { for all }|x|>R_{\varepsilon} .
$$

Observe now that by (3.3) and Lipschitz continuity

$$
\bar{u}(x, t) \geqslant \bar{u}(0, t)-\|\nabla \bar{u}\|_{\infty}|x|=\sqrt{t} \bar{u}(0,1)-\|\nabla \bar{u}\|_{\infty}|x| .
$$

Since $\bar{u}(0,1)>0$ by Lemma 4.1 , there exists $t_{\varepsilon}>0$ sufficiently large such that

$$
\bar{u}(x, t) \geqslant u_{0}(x) \quad \text { for all }|x| \leqslant R_{\varepsilon}, t \geqslant t_{\varepsilon} .
$$

Therefore, by (4.4), (4.5), and by comparison we get that for every $t>0$

$$
u(x, t) \leqslant \bar{u}\left(x, t+t_{\varepsilon}\right)+\varepsilon \quad \forall t \geqslant 0, x \in \mathbb{R}^{N} .
$$

By (4.6) and (4.3) we get that for $\varepsilon>0, \delta>0$

$$
\bar{u}\left(x, t-t_{\delta}\right)-\bar{u}(x, t)-\delta \leqslant u(x, t)-\bar{u}(x, t) \leqslant \bar{u}\left(x, t+t_{\varepsilon}\right)-\bar{u}(x, t)+\varepsilon .
$$

We conclude sending $t \rightarrow+\infty$, and recalling Theorem 3.1. In case that $\nabla^{2} \varphi, \psi$ are more regular, we conclude recalling Remark 3.2.

\section{StABiLity OF HYPERPLANES}

In this section we show that hyperplanes are stable with respect to the flow (1.1), if the anisotropy is regular, that is, (2.2) holds. In particular we show that if the initial datum is flat at infinity, then the solution stabilizes to the hyperplane at which the initial datum is asymptotic. The same result has been obtained in the isotropic 
case by integral estimates and comparison with large balls in $[9,11]$, and by using the heat kernel, in dimension 2, in [19]. Here we provide a different proof based on construction of suitable periodic barriers.

We start with a preliminary lemma on 1-dimensional periodic barriers.

Lemma 5.1. Assume $(2.2)$, and let $f_{0}: \mathbb{R} \rightarrow \mathbb{R}$ be a function of class $C^{1,1}(\mathbb{R})$ which is $\mathbb{Z}$-periodic and odd. Let $u(x, t)$ be the solution to $(2.7)$ with initial datum $u_{0}(x):=f_{0}\left(x \cdot e_{1}\right)$. Then

$$
\lim _{t \rightarrow+\infty} u(x, t)=0 \quad \text { uniformly in } C\left(\mathbb{R}^{N}\right) .
$$

Proof. By uniqueness we have that

$$
u(x, t)=f\left(x \cdot e_{1}, t\right)
$$

where $f: \mathbb{R} \times[0,+\infty) \rightarrow \mathbb{R}$ satisfies $f(r, 0)=f_{0}(r), f(r, t)=-f(-r, t)$ and $f(r+z, t)=f(r, t)$ for every $z \in \mathbb{Z}$. Notice also that by Proposition $2.9 u$ is globally Lipschitz continuous in $x, t$. Differentiating in time the anisotropic perimeter of the graph of $u$, for all $t>0$ we get that

$$
\begin{aligned}
\int_{[0,1]^{N}} \varphi\left(-\nabla u_{0}(x), 1\right) \mathrm{d} x & \geq \int_{[0,1]^{N}} \varphi\left(-\nabla u_{0}(x), 1\right) \mathrm{d} x-\int_{[0,1]^{N}} \varphi(-\nabla u(x, t), 1) \mathrm{d} x \\
& =-\int_{0}^{t} \int_{[0,1]^{N}} u_{t} \operatorname{div}(\nabla \varphi((-\nabla u, 1) \mathrm{d} x \mathrm{~d} s \\
& =\int_{0}^{t} \int_{[0,1]^{N}} \frac{u_{t}^{2}}{\psi(-\nabla u, 1)} \mathrm{d} x \mathrm{~d} s
\end{aligned}
$$

which implies that

$$
\int_{0}^{+\infty} \int_{0}^{1} f_{t}^{2} \mathrm{~d} x \mathrm{~d} s=\int_{0}^{+\infty} \int_{[0,1]^{N}} u_{t}^{2} \mathrm{~d} x \mathrm{~d} s \leq C
$$

where the constant

$$
C:=\left(\max _{|\xi| \leq \max _{[0,1]} f_{0}^{\prime}} \psi(\xi, 1)\right) \int_{[0,1]^{N}} \varphi\left(-\nabla u_{0}(x), 1\right) \mathrm{d} x
$$

depends only on the initial function $f_{0}$. It follows that there exists a sequence of times $t_{n} \rightarrow+\infty$ such that $f_{t}\left(\cdot, t_{n}\right) \rightarrow 0$ in $L^{2}([0,1])$ as $n \rightarrow+\infty$. Recalling Proposition 2.9, up to extracting a further subsequence we can also assume that $u\left(x, t_{n}\right) \rightarrow \bar{u}(x)$ uniformly in $C^{1}\left(\mathbb{R}^{N}\right)$ as $n \rightarrow+\infty$, where $\bar{u}(x)=\bar{f}\left(x \cdot e_{1}\right)$ with $\bar{f} \mathbb{Z}$-periodic.

Evaluating now (2.7) at $t=t_{n}$, after an integration by parts we get that

$$
\int_{[0,1]^{N}} \frac{u_{t}\left(x, t_{n}\right)}{\psi\left(-\nabla u\left(x, t_{n}\right), 1\right)} \eta\left(x \cdot e_{1}\right) \mathrm{d} x=\int_{[0,1]^{N}} \nabla \varphi\left(-\nabla u\left(x, t_{n}\right), 1\right) \cdot e_{1} \eta^{\prime}\left(x \cdot e_{1}\right) \mathrm{d} x
$$

for all $\eta \in C^{1}(\mathbb{R}) \mathbb{Z}$-periodic. Passing to the limit as $n \rightarrow+\infty$, we finally get that the function $\bar{u}$ satisfies

$$
\int_{[0,1]^{N}} \nabla_{x} \varphi(-\nabla \bar{u}(x), 1) \cdot e_{1} \eta^{\prime}\left(x \cdot e_{1}\right) \mathrm{d} x=0
$$


for all $\eta$, that is, $\bar{u}$ is a periodic, odd and smooth solution to the anisotropic minimal surface equation

$$
\operatorname{div}\left(\nabla_{x} \varphi(-\nabla \bar{u}, 1)\right)=0
$$

Recalling that we are assuming (2.2), we get that by the strong maximum principle, and the periodicity of $\bar{u}$, we conclude that $\bar{u} \equiv 0$.

Theorem 5.2. Let $E_{0} \subseteq \mathbb{R}^{N+1}$ such that $\partial E_{0}$ is a Lipschitz surface and assume that there exists a half-space $H$ for which

$$
\lim _{R \rightarrow+\infty} d_{H}\left(E_{0} \backslash B(0, R), H \backslash B(0, R)\right)=0
$$

where $d_{H}(A, B)$ is the Hausdorff distance between the sets $A, B$. Then we have

$$
\lim _{t \rightarrow+\infty} d_{H}\left(E_{t}, H\right)=0
$$

Proof. We may assume without loss of generality that $H=\left\{(x, z) \in \mathbb{R}^{n} \times \mathbb{R} \mid z \leqslant 0\right\}$. Moreover, by the assumption that $\lim _{R \rightarrow+\infty} d\left(E_{0} \backslash B(0, R), H \backslash B(0, R)\right)=0$, there exist two Lipschitz functions $u_{0}, v_{0}: \mathbb{R}^{N} \rightarrow \mathbb{R}$ such that $\lim _{|x| \rightarrow+\infty} u_{0}(x)=0=\lim _{|x| \rightarrow+\infty} v_{0}(x)$ and $\left\{(x, z) \in \mathbb{R}^{n} \times \mathbb{R} \mid z \leqslant u_{0}(x)\right\} \subseteq E_{0} \subseteq\left\{(x, z) \in \mathbb{R}^{n} \times \mathbb{R} \mid z \leqslant\right.$ $\left.v_{0}(x)\right\}$. By comparison we get that $\left\{(x, z) \in \mathbb{R}^{n} \times \mathbb{R} \mid z \leqslant u(x, t)\right\} \subseteq E_{t} \subseteq\left\{(x, z) \in \mathbb{R}^{n} \times \mathbb{R} \mid z \leqslant v(x, t)\right\}$, where $u(x, t), v(x, t)$ are the solutions to $(2.7)$ with initial datum $u_{0}, v_{0}$.

We claim that $\lim _{t \rightarrow+\infty} u(x, t)=0=\lim _{t \rightarrow+\infty} v(x, t)$ uniformly. If the claim is true, then the result follows.

It is sufficient to prove the claim only for $u$ (since for $v$ is completely analogous), moreover, we restrict to the case in which $u_{0} \geqslant 0$ (or equivalenty $u_{0} \leqslant 0$ ). Indeed the general case is easily obtained by using as barriers the solutions with initial data $u_{0}^{+}=\max \left(u_{0}, 0\right)$ and $u_{0}^{-}=\min \left(u_{0}, 0\right)$.

So, we prove the claim only for the case $u_{0} \geqslant 0$. Note that by comparison, since the constants are stationary solutions, $0 \leqslant u(x, t) \leqslant \max u_{0}$ for all $x \in \mathbb{R}^{N}, t>0$.

First of all we prove that

$$
\inf _{x} u(x, t)=0
$$

for all $t>0$, by comparison with shrinking Wulff shapes as constructed in Remark 2.6. Indeed for every $\varepsilon>0$, let us fix $R>0$ such that $u_{0}(x) \leqslant \varepsilon$ for all $|x| \geqslant R$. Let $\tilde{E}_{0}$ be the subgraph of $\max \left(u_{0}, \varepsilon\right)$. Note that for $|x| \geqslant R$, $\partial \tilde{E}_{0}$ is a hyperplane, and then at every $|x|>R$, there exists $R_{|x|}>0$, such that $R_{|x|} \rightarrow+\infty$ as $|x| \rightarrow+\infty$ and that $\tilde{E}_{0}$ satisfies at $x$ the exterior $R_{|x|} W_{\varphi^{0}}$ condition. So, arguing as in the proof of Theorem 3.1, we get that for every $t>0$, there exists $K>0$ such that $|u(x, t)| \leqslant 2 \varepsilon$ for $|x|>K$. This gives the desired property $\inf _{x} u(x, t)=0$.

Now we note that by comparison, $M(t)=\sup _{x} u(x, t)$ is decreasing. We define $0 \leqslant \bar{M}:=\lim _{t \rightarrow+\infty} M(t)=$ $\inf _{t} M(t)$. Now we claim that

$$
\bar{M}=0 .
$$

If the claim holds, then we get the conclusion.

Assume by contradiction that $\bar{M}>0$. We fix $0<\varepsilon<\frac{\bar{M}}{2}$ and $\bar{t}>0$ such that $M(\bar{t}) \leqslant \bar{M}+\varepsilon$. We fix also $R=R(\bar{t})$ such that $u(x, \bar{t})<\frac{\bar{M}}{2}$ for all $|x|>R$. Now we shall get a contradiction by constructing a periodic barrier as in Lemma 5.1 (up to suitable vertical translations). We fix a smooth even function $f_{0}:[-2 R, 2 R] \rightarrow \mathbb{R}$, such that $f_{0}(z)=\bar{M}+\varepsilon$ for $z \in[-R, R], f_{0}(-2 R)=f_{0}(2 R)=\frac{3}{4} \bar{M}+\frac{\varepsilon}{2}$ and $f_{0}(z)$ is increasing in $(-2 R,-R)$ 
and decreasing in $(R, 2 R)$. Now we extend it to a function $f_{0}:[-2 R, 6 R] \rightarrow \mathbb{R}$ by putting

$$
f_{0}(z+2 R)=-f_{0}(-z+2 R)+\frac{3}{2} \bar{M}+\varepsilon
$$

Note that $f_{0}(z+2 R)-\frac{3}{4} \bar{M}-\frac{\varepsilon}{2}$ is an odd function. Finally, we extend it by periodicity to be a $8 R \mathbb{Z}$ periodic function.

Let $v_{0}(x):=f_{0}\left(x \cdot e_{1}\right)$, and note that by construction, $u(x, \bar{t}) \leqslant v_{0}(x)$ for all $x \in \mathbb{R}^{N}$ and then by comparison

$$
u(x, t+\bar{t}) \leqslant v(x, t), \quad \text { and in particular } \limsup _{t \rightarrow+\infty} u(x, t) \leqslant \lim _{t \rightarrow+\infty} v(x, t)
$$

where $v(x, t)$ is the solution to (2.7) with initial datum $v_{0}$. Now by Lemma 5.1 we get that $\lim _{t \rightarrow+\infty} v(x, t)=$

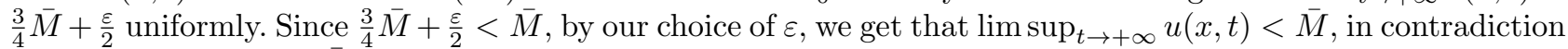
with the definition of $\bar{M}$.

\section{REFERENCES}

[1] B. Andrews, Volume-preserving anisotropic mean curvature flow. Indiana Univ. Math. J. 50 (2001) $783-827$.

[2] G. Barles and P.E. Souganidis, A new approach to front propagation problems: theory and applications. Arch. Ratl. Mech. Anal. 141 (1998) 237-296.

[3] G. Bellettini, V. Caselles, A. Chambolle and M. Novaga, Crystalline mean curvature flow of convex sets. Arch. Ratl. Mech. Anal. 179 (2006) 109-152.

[4] H. Brézis, Opérateurs maximaux monotones et semi-groupes de contractions dans les espaces de Hilbert, North-Holland Mathematics Studies, No. 5, North-Holland Publishing Co., Amsterdam-London; American Elsevier Publishing Co., Inc., New York (1973).

[5] A. Cesaroni and M. Novaga, Fractional mean curvature flow of Lipschitz graphs. Preprint arxiv:2103.11346 (2021).

[6] A. Chambolle, M. Morini, M. Novaga and M. Ponsiglione, Existence and uniqueness for anisotropic and crystalline mean curvature flows. J. Am. Math. Soc. 32 (2019) 779-824.

[7] A. Chambolle, M. Morini, M. Novaga and M. Ponsiglione, Generalized crystalline evolutions as limits of flows with smooth anisotropies. Anal. PDE 12 (2019) 789-813.

[8] A. Chambolle, M. Morini and M. Ponsiglione, Existence and uniqueness for a crystalline mean curvature flow. Commun. Pure Appl. Math. 70 (2017) 1084-1114.

[9] J. Clutterbuck and O.C. Schn'urer, Stability of mean convex cones under mean curvature flow. Math. Z. 267 (2011) $535-547$.

[10] K. Ecker and G. Huisken, Mean curvature evolution of entire graphs. Ann. Math. (2) 130 (1989) 453-471.

[11] K. Ecker, Regularity theory for mean curvature flow. Vol. 57 of Progress in Nonlinear Differential Equations and their Applications, Birkhäuser Boston, Inc., Boston, MA (2004).

[12] M.-H. Giga, Y. Giga and H. Hontani, Self-similar expanding solutions in a sector for a crystalline flow. SIAM J. Math. Anal. 37 (2005) 1207-1226.

[13] Y. Giga, Surface evolution equations. Vol. 99 of Monographs in Mathematics. Birkhäuser Verlag, Basel (2006). A level set approach.

[14] Y. Giga and N. Požár, Approximation of general facets by regular facets with respect to anisotropic total variation energies and its application to crystalline mean curvature flow. Commun. Pure Appl. Math. 71 (2018) 1461-1491.

[15] Y. Giga and N. Požár, A level set crystalline mean curvature flow of surfaces. Adv. Differ. Equ. 21 (2016) $631-698$.

[16] G. Huisken, Flow by mean curvature of convex surfaces into spheres. J. Differ. Geom. 20 (1984) $237-266$.

[17] G.M. Lieberman, Second order parabolic differential equations. World Scientific Publishing Co., Inc., River Edge, NJ (1996).

[18] A. Lunardi, Analytic semigroups and optimal regularity in parabolic problems. Modern Birkhäuser Classics, Birkhäuser/Springer Basel AG, Basel (1995).

[19] M. Nara and M. Taniguchi, The condition on the stability of stationary lines in a curvature flow in the whole plane. J. Differ. Equ. 237 (2007) 61-76. 
[20] N.S. Trudinger, On regularity and existence of viscosity solutions of nonlinear second order, elliptic equations. Vol. 2 of Progr. Nonlinear Differ. Equ. Appl.. Birkhäuser Boston, Boston, MA (1989).

[21] L. Wang, A Bernstein type theorem for self-similar shrinkers. Geom. Dedicata 151 (2011) 297-303.

\section{Subscribe to Open (S2O) A fair and sustainable open access model}

This journal is currently published in open access under a Subscribe-to-Open model ( $\mathrm{S} 2 \mathrm{O}$ ). S2O is a transformative model that aims to move subscription journals to open access. Open access is the free, immediate, online availability of research articles combined with the rights to use these articles fully in the digital environment. We are thankful to our subscribers and sponsors for making it possible to publish this journal in open access, free of charge for authors.

\section{Please help to maintain this journal in open access!}

Check that your library subscribes to the journal, or make a personal donation to the S2O programme, by contacting subscribers@edpsciences.org

More information, including a list of sponsors and a financial transparency report, available at: https://www.edpsciences.org/en/maths-s2o-programme 